\title{
Ultrafast laser inscribed cladding waveguides in Nd:YAG crystal for mid-infrared wavelength
}

\author{
Qiang An ${ }^{a}$, Yuechen Jia ${ }^{a}$, Hongliang Liu ${ }^{a}$, \\ Javier Rodríguez Vázquez de Aldana ${ }^{\mathrm{b}}$, Feng Chen ${ }^{\mathrm{a}, *}$ \\ a School of Physics, State Key Laboratory of Crystal Materials and Key Laboratory of Particle Physics and Particle Irradiation, Ministry of Education, Shandong \\ University, Jinan 250100, China \\ ${ }^{\mathrm{b}}$ Laser Microprocessing Group, Universidad de Salamanca, Salamanca 37008, Spain
}

\section{A R T I C L E I N F O}

\section{Article history:}

Received 29 July 2013

Received in revised form

7 September 2013

Accepted 10 September 2013

Available online 8 October 2013

Keywords:

Cladding waveguide

Nd:YAG

Mid-infrared

\begin{abstract}
A B S T R A C T
We report on tubular cladding optical waveguides fabricated in neodymium doped yttrium aluminum garnet (Nd:YAG) crystal by ultrafast laser inscription. The structure can support guidance at the wavelength of $4 \mu \mathrm{m}$ for the TE and TM polarizations. The refractive index contrasts of the cladding waveguides for the two transverse polarizations were estimated to be $\Delta n_{T E} \approx-7.5 \times 10^{-3}$ and $\Delta n_{T M} \approx-6.5 \times 10^{-3}$. The propagation losses of the cladding waveguides at the wavelength of $4 \mu \mathrm{m}$ were determined to be as low as $\sim 0.7 \mathrm{~dB} / \mathrm{cm}$ for the TE polarization and $\sim 1.0 \mathrm{~dB} / \mathrm{cm}$ for the TM polarization.
\end{abstract}

(c) 2013 Elsevier Ltd. All rights reserved.

\section{Introduction}

Nowadays, the ultrafast laser inscription (ULI), as one of the most powerful and unique techniques for three-dimensional (3-D) volume micro-structuring of transparent dielectrics because of its outstanding advantages of 3-D fast and versatile processing, has been widely employed to fabricate optical waveguides in a large number of optical materials, involving glasses [1-3], optical crystals [4-7], ceramics [8-11], and polymers [12]. The focused ultrafast laser in transparent crystal can produce some controlled micro-modifications in the direct-irradiate region, forming the socalled "track" or "filament", and induce a positive or negative change of the refractive index in the focal volume [9]. To the best of our knowledge, in most of the crystals, a reduction of the refractive index in the focal volume could be induced after the scanning of the ultrafast laser. So the materials fabricated by the ULI technique can form the waveguide structure in a few crystals. The ULI waveguides could be divided into four categories: Type I waveguides with single line (an increase of the refractive index in the focal volume), Type II waveguides with double-line filaments (a decrease of the refractive index in the filaments fabricated by the scanning of the ultrafast laser), Type III cladding waveguides with large area of cross sections (a decrease of the refractive index in the damage track induced by the ultrafast laser) and Type IV

\footnotetext{
*Corresponding author. Tel.: +86531 88363007; fax: +8653188363350.

E-mail address: drfchen@sdu.edu.cn (F. Chen).
}

ridge waveguide ablated by the ULI technique on the planar waveguide [13]. Concerning the cladding waveguides, it includes the low-index tubular cladding structure with a large number of damage tracks and the relatively high-index core waveguide region surrounded by the filaments. In theory, we can adjust the shape and size of the cladding waveguide structure with more flexibility [14-16]. Thus, the waveguide structure with the special shape and size can be used to couple into or out of the certain fiber with the high efficiency. Moreover, the light from visible to midinfrared (MIR) range till longer wavelength regions can be guided in the cladding waveguide structure due to its large dimensions. In $\mathrm{Nd}$, Yb or Tm doped YAG ceramics or crystals, $\mathrm{Nd}: \mathrm{YVO}_{4}, \mathrm{BiB}_{3} \mathrm{O}_{6}$, $\mathrm{KTiOPO}_{4}$ and $\mathrm{ZnS}$ crystals, such cladding waveguide structures have been achieved [13,17-21]. Especially, the ultrafast laser inscribed cladding waveguides at MIR wavelengths in Tm doped YAG ceramic and ZnS crystal have been reported [18,21].

Accompanying the development of Type-II quantum well lasers (based on GaSb and dilute-nitride semiconductors) and InP-based quantum cascade lasers [22-25], the MIR spectral region has become vitally fascinating because of the various charming applications, for example, optical sensing, thermal imaging, biomedicine, free-space communications, etc. [26]. In particular, it is well known that in the MIR region the two atmospheric transmission windows (3-5 and $8-14 \mu \mathrm{m}$ ) are the most important spectral regime for modern optical communication. The MIR wavelength included in the two windows region can be propagated with high transmission, low reflection and less absorption. Besides, as the basic components for the integrated photonics, the waveguide 
structure have been used widely in the modern optical communication because the special structure of the waveguide can confine light propagation in reduced volumes; thus reaching high optical intensities of light. Furthermore, the cladding waveguide structure with large dimension can support guidance at the MIR wavelength and enhance optical properties of the bulk materials. Based on such structure, we can realize a number of MIR applications in chip-scale wafers which can be applied in the modern optical communication. And some waveguide structures in siliconon-insulator [27,28], silica on silicon [29], and chalcogenide glass [30] at the MIR range have been reported.

Recently, the active ions (e.g., $\mathrm{Nd}, \mathrm{Yb}, \mathrm{Er}, \mathrm{Tm}, \mathrm{Cr}$ and $\mathrm{Ho}$ ) doped crystals, glasses and ceramics were widely applied in the laser pumping. And the active ions doped yttrium aluminum garnet crystal $\left(\mathrm{Y}_{3} \mathrm{Al}_{5} \mathrm{O}_{12}\right.$ or $\left.\mathrm{YAG}\right)$ is one of the most used and researched materials owing to its excellent optical and physical properties, such as outstanding fluorescence, mechanical, thermal properties and a transparency window covering from visible to infrared wavelengths $(0.4-5.5 \mu \mathrm{m})$ [31], which can be used in the MIR laser pumping. As is well known, the active Nd ions doped in the crystal do not affect the guiding properties of the waveguide. By investigating the MIR guiding properties of Nd:YAG crystal, one could explore the possibility of the MIR laser generation in suitable rare-earth ion (such as $\mathrm{Er}$, Tm, Ho etc.) doped YAG systems. For example, Ho and Tm co-doped YAG crystal could be applied for $\sim 2 \mu \mathrm{m}$ laser pumping from the visible light [32]. The cladding waveguide structure fabricated by the ULI technique in the Nd: YAG ceramic and single crystal had been reported by Liu and Okhrimchuk [33,34]. In this work, to our best knowledge for the first time, we report on the guiding properties of optical cladding waveguides at the wavelength of $4 \mu \mathrm{m}$ in Nd:YAG crystal fabricated by the ULI technique.

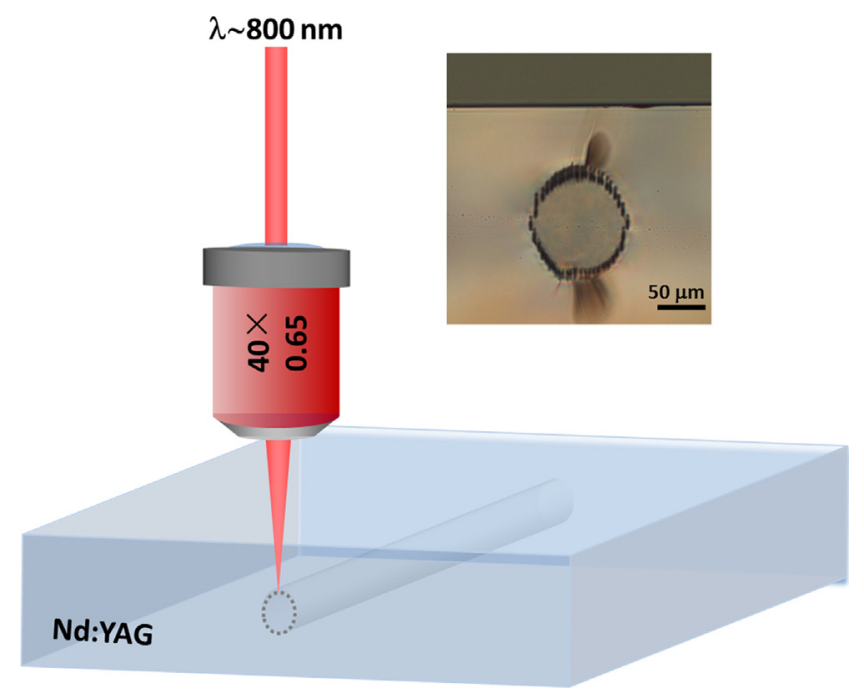

Fig. 1. The fabrication schematic of Nd:YAG cladding waveguide by ultrafast laser inscription. The inset shows the photograph of cross section of the cladding waveguide in Nd:YAG crystal by an optical microscope (Axio Imager, Carl Zeiss) operating in transmission mode.

\section{Experimental details}

The Nd:YAG crystal used in our work was cut to the dimensions of $9(x) \times 10(y) \times 2.0(z) \mathrm{mm}^{3}$, and the largest and the two edge faces of the sample were polished to an optical quality. The cladding waveguide structures were fabricated by the amplified Ti:sapphire laser system of the Universidad de Salamanca, Spain. The fabrication schematic is depicted in Fig. 1. The laser system delivered $120 \mathrm{fs}$ pulses, linearly polarized at the wavelength of $800 \mathrm{~nm}$, with a repetition rate of $1 \mathrm{kHz}$ and $1 \mathrm{~mJ}$ maximum pulse energy. A calibrated neutral density filter and a set of half-wave plate and linear polarizer were used to reduce the pulse energy to $0.6 \mu \mathrm{J}$. The beam was then focused $150 \mu \mathrm{m}$ beneath the sample surface by employing a microscope objective (Leica $40 \times$, numerical aperture N.A. $=0.65$ ), while the sample was moved at a constant velocity of $700 \mu \mathrm{m} / \mathrm{s}$ (along the $y$ direction) with the help of a motorized 3-axes stage. As a result, a damage track along the crystal was produced. The process was repeated at certain depths (with the maximum of $150 \mu \mathrm{m}$ ) and positions of the sample in order to produce a tubular structure formed by multiple parallel damage tracks. The separation between adjacent tracks was set to $3 \mu \mathrm{m}$. Consequently, a cladding waveguide structure including the low-index tubular structure with a large number of damage tracks and the relatively high-index core waveguide region surrounded by the filaments was achieved. In the inset of Fig. 1, we show a picture of the waveguide taken with an optical microscope (Axio Imager, Carl Zeiss) operating in transmission mode.

In order to investigate the near-field modal profiles of the cladding waveguide, we employed the end-face coupling arrangement which also can determine the value of the maximum refractive index contrast, as shown in Fig. 2. According to the above-mentioned results, the refractive index distribution can be reconstructed. In the end-face coupling arrangement, the sample was mounted on a 6-axes optical stage. The incident light at the wavelength of $4 \mu \mathrm{m}$, generated in the Tunable Laser System - MIR ${ }^{\mathrm{TM}} 8025$ (Daylight Solutions, Inc.), was focused by a MIR microscope objective lens (ZnSe, LFO-5-12-3.75, N.A. $=0.13$ ) placed after a linear polarizer, and then coupled into the cladding waveguide. Afterwards, the output light was collected by using another MIR microscope objective lens at the other facet of the sample. The near field intensity distributions were recorded with a MIR charge coupled device (CCD) experimentally. And the power of the incident and output light was measured by the optical power meter (Model 1916-R, Newport), and the total loss of the waveguide system (including coupling loss and propagation loss) was obtained. The coupling efficiency was calculated by the size of overlap area between the incident light and the waveguide modes. By subtracting coupling loss and the Fresnel deflection (occurring at the two aircrystal end facets) from the total loss, we could approximately calculate the propagation losses of the cladding waveguide structure. We also measured the N.A. of the waveguide in order to get the maximum value of refractive index changes of the waveguide at the wavelength of $4 \mu \mathrm{m}$, by adjusting the position of the incident coupled light.

\section{Results and discussion}

The inset of Fig. 1 shows the photograph of the cross section of the cladding waveguide in the Nd:YAG crystal by using an optical

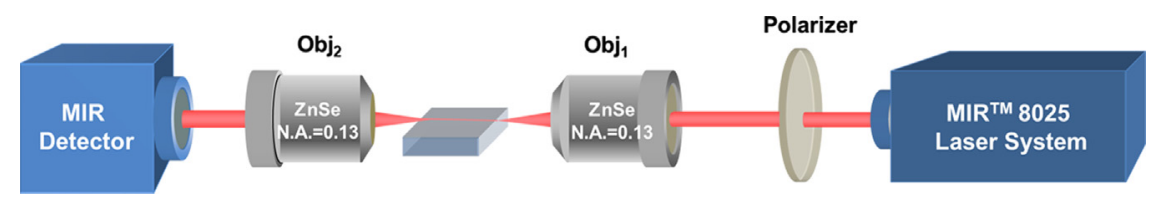

Fig. 2. Schematic of the end-face coupling arrangement used to investigate the optical properties of the cladding waveguide in Nd:YAG crystal. 
microscope operating in transmission mode. As we can see from the figure, the diameter of the cladding structure is $\sim 100 \mu \mathrm{m}$. The core region of the waveguide structure is located inside the circle that consists of a large number of filaments induced by the ultrafast laser inscription. Some cracks of the crystal that do not affect the core of the fabricated structure can be seen in the picture. They are produced by multiple irradiation of the sample during the fabrication procedure.

Due to the geometry of the cladding waveguides, there is no way to directly measure the refractive index profile by the typical measuring method, such as the m-line technology. However, the maximum value of the waveguide refractive index contrast can be obtained by the measurement of the N.A. of the waveguide, which could be roughly approximated by utilizing the equation

$\Delta n \approx \frac{\sin ^{2} \Theta_{m}}{2 n}$

where $\Theta_{m}$ is the maximum incident angular deflection angle where no transmitted light is coupling into the cladding waveguide, while $n$ is the refractive index of the substrate [5]. Based on a

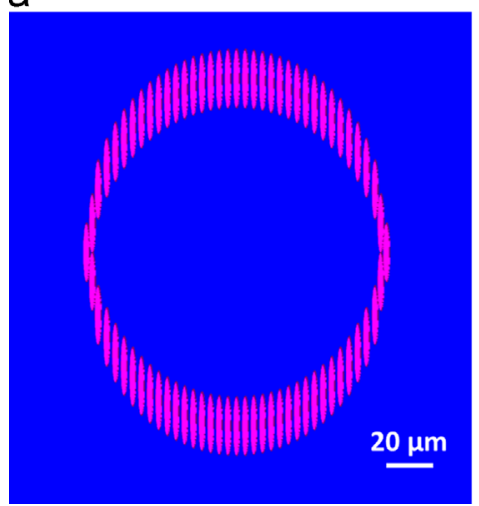

b

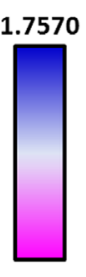

1.7495

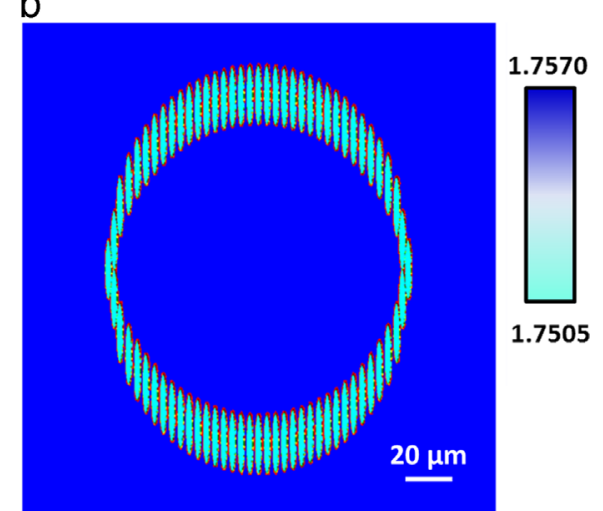

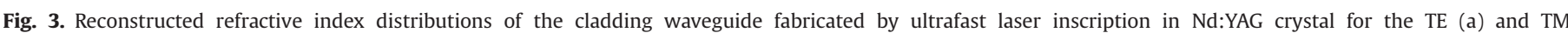
(b) polarizations.
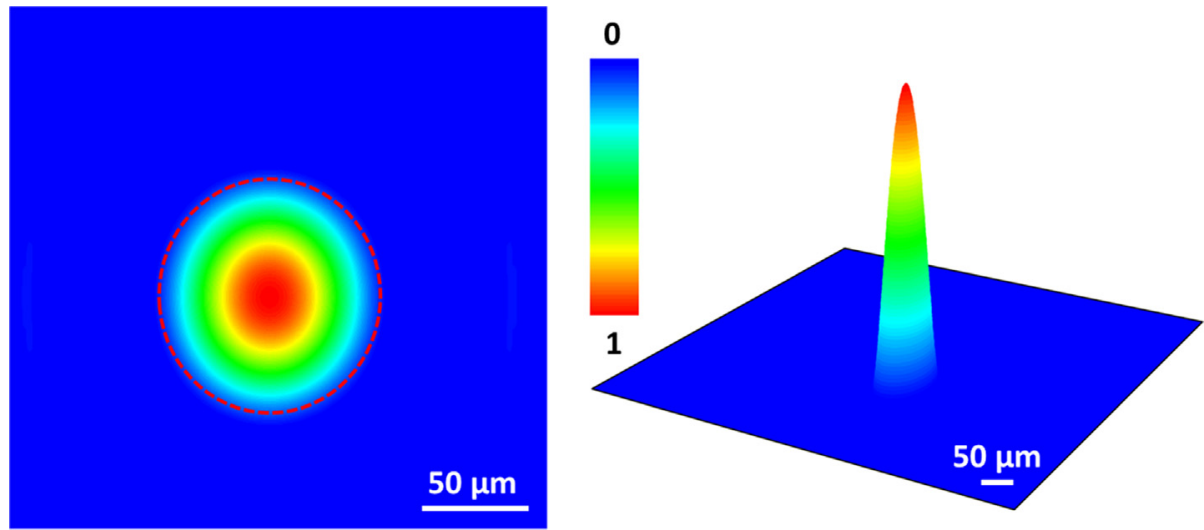

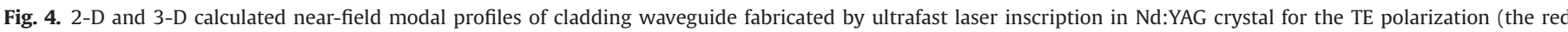

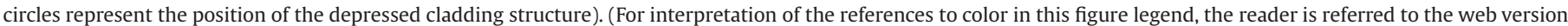
of this article.)
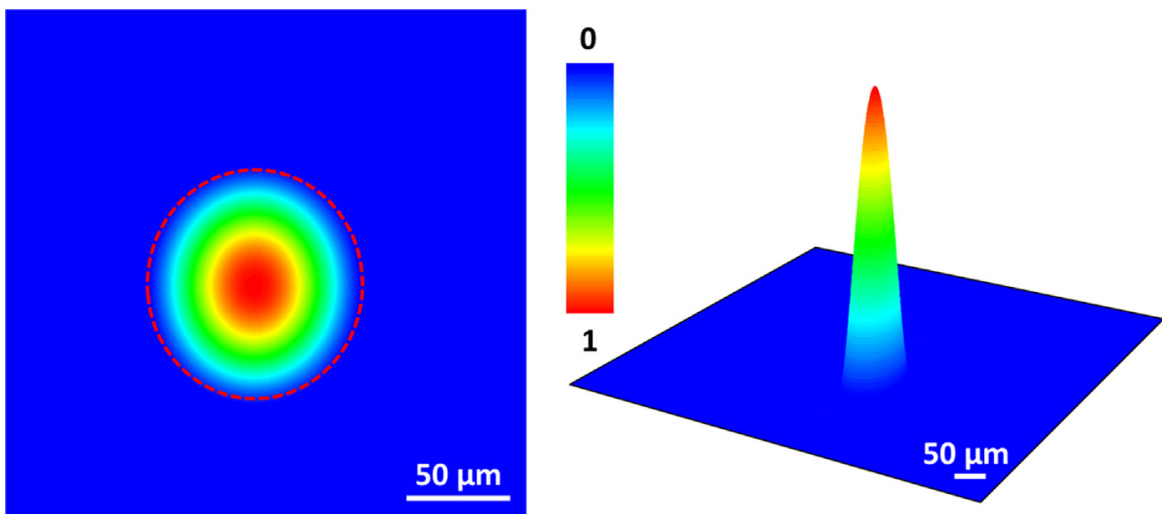

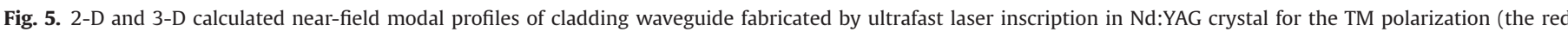

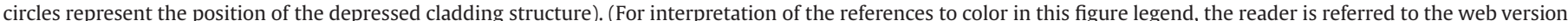
of this article.) 

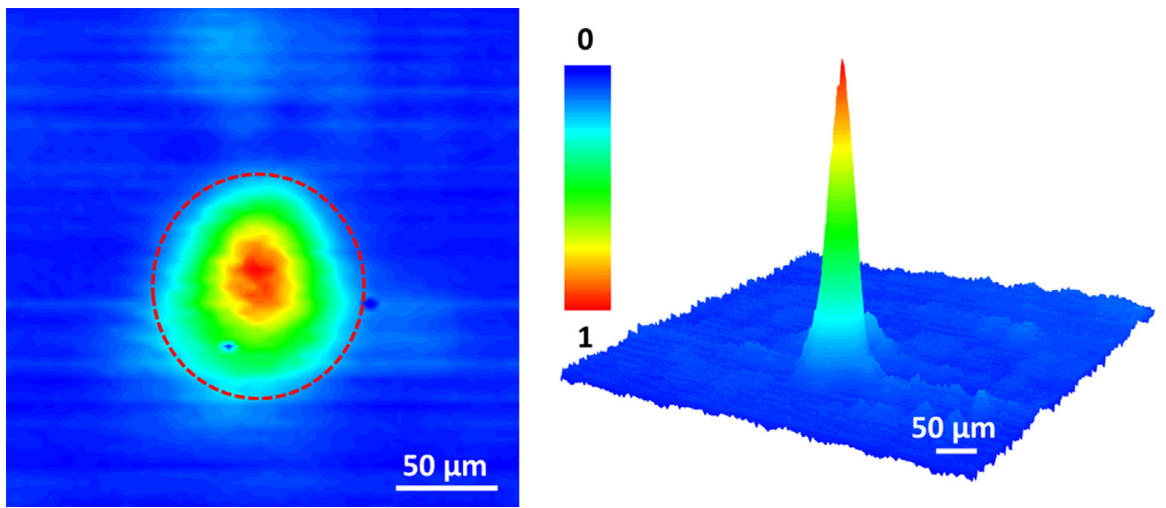

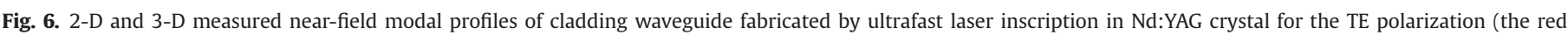

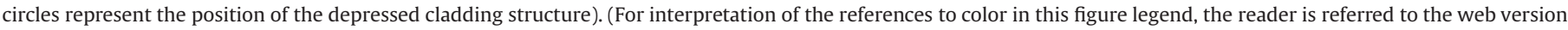
of this article.)
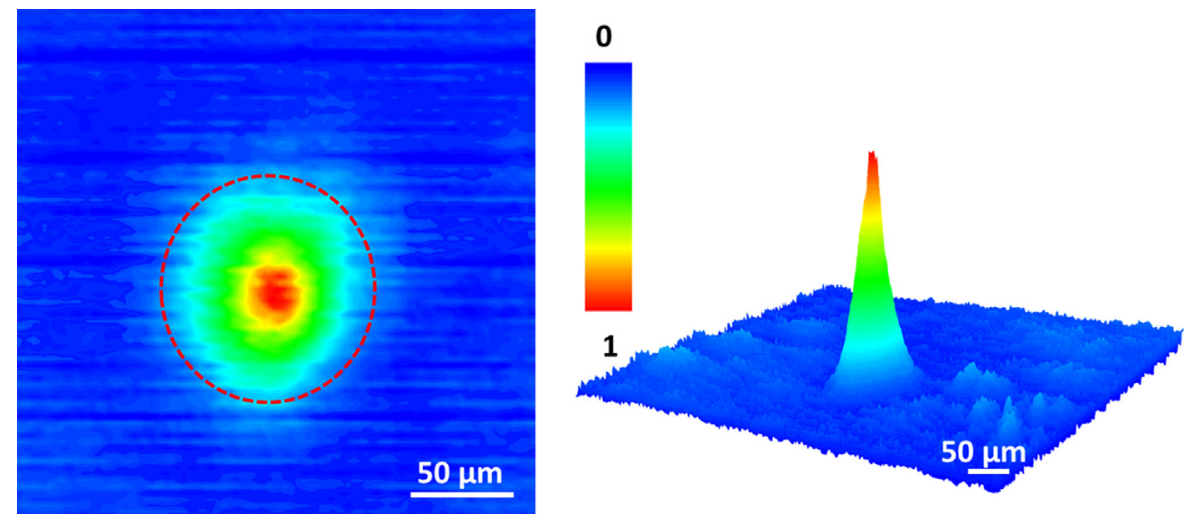

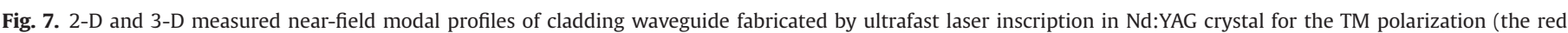

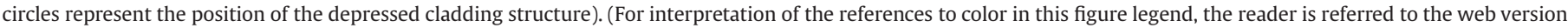
of this article.)

the measured $\Theta_{m}$, we estimated the refractive index contrast to be $\Delta n_{T E} \approx-7.5 \times 10^{-3}$ and $\Delta n_{T M} \approx-6.5 \times 10^{-3}$ between the lowindex tubular cladding structure formed by the inscribed filaments, and the core waveguide region. According to the refractive index contrast, we roughly reconstructed the refractive index distribution for the TE and TM polarization of the cladding waveguide, respectively, as shown in Fig. 3(a) and (b). The two profiles obtained from RSoft $\odot$ software can roughly display the shape and size of the cladding waveguide [35]. And we can also see that the change in the refractive index for the TE polarization is larger than that for the TM polarization. Based on the two refractive index distributions, the 2-D and 3-D calculated near-field modal profiles for the TE and TM polarizations, shown in Figs. 4 and 5, was achieved by the BeamPROP module of the RSoft $@$ software which is the most widely used propagation technique for modeling integrated and fiber optical photonic devices.

In order to investigate the waveguide properties, the end-face coupling arrangement was employed to measure the near-field modal profiles of the cladding waveguide in the Nd:YAG crystal for both the TE and TM polarizations at the wavelength of $4 \mu \mathrm{m}$. It is clear from Figs. 6 and 7 that the fabricated cladding waveguide structure supports single mode light of $4 \mu \mathrm{m}$ for both TM and TE polarizations. And the calculated near-field modal profiles for the TE and TM polarizations are in very good agreement with the experimental results shown in Figs. 6 and 7, respectively. It also implies that the reconstructed refractive index distributions were acceptable and the simulations based on the reconstructed refractive index profile were successful. Afterwards, we measured the incident and output light power by using the optical power meter working at the MIR region. By computation, we achieved the effective incident light power and then approximately calculated the propagation loss for the TE and TM polarizations at the wavelength of $4 \mu \mathrm{m}, \sim 0.7 \mathrm{~dB} / \mathrm{cm}$ and $\sim 1.0 \mathrm{~dB} / \mathrm{cm}$, respectively. Obviously, compared to the TM modal light, the cladding waveguide can propagate the light for the TE polarization better. We think that two possible factors might be responsible for such difference, just as our previously reported cladding waveguide structures in zinc sulfide crystal [21]. On one hand, there is a track separation of $3 \mu \mathrm{m}$ between the damage filaments, so that the light with the TM polarization (parallel to these damage tracks) could scatter out of the cladding waveguide structure easier than the light for the TE polarization. On the other hand, the measured refractive index contrast of the structures is different for both polarizations. For the TE polarization, the refractive index change is larger and it may confine the light inside the cladding structure better.

An improvement of the optical properties of the cladding waveguides in Nd:YAG or other crystal could be achieved, in principle, by reducing the scan separation and by increasing the pulse energy during the irradiation (just to increase the refractive index contrast). However, both factors are limited by the damage produced in the crystal, which could lead to severe cracks affecting the core region of the waveguide.

\section{Conclusion}

In summary, we have reported the fabrication of cladding waveguides in Nd:YAG crystal by using the ultrafast laser inscription 
technique. The guiding properties at the wavelength of $4 \mu \mathrm{m}$ have been investigated, denoting single mode behavior for both TE and TM polarizations. The measured propagation losses were $\sim 0.7 \mathrm{~dB} /$ $\mathrm{cm}$ for the TE polarization and $\sim 1.0 \mathrm{~dB} / \mathrm{cm}$ for the TM polarization. The cladding waveguide structure with better optical performance could be achieved by reducing the lateral separation and increasing the refractive index change contrast. Some potential low-loss devices of the active ions (e.g., Er, Dy, Ho, or Tm) doped YAG cladding waveguides can be fabricated by the ultrafast laser inscription technology and applied in MIR optical communications or MIR laser pumping.

\section{Acknowledgments}

The work is supported by the National Natural Science Foundation of China (Nos. 11274203 and 11111130200), the Spanish Ministerio de Ciencia e Innovación (CSD2007-00013 and FIS2009-09522) and the Junta de Castilla y León (Project SA086A12-2).

\section{References}

[1] Psaila ND, Thomson RR, Bookey HT, Kar AK, Chiodo N, Osellame R, et al. Er:Ybdoped oxyfluoride silicate glass waveguide amplifier fabricated using femtosecond laser inscription. Applied Physics Letters 2007:90:131102.

[2] Little DJ, Ams M, Dekker P, Marshall GD, Dawes JM, Withford MJ. Femtosecond laser modification of fused silica: the effect of writing polarization on $\mathrm{Si}-\mathrm{O}$ ring structure. Optics Express 2008:16:20029-37.

[3] Lancaster DG, Gross S, Ebendorff-Heidepriem H, Kuan K, Monro TM, Ams M, et al. Fifty percent internal slope efficiency femtosecond direct-written $\mathrm{Tm}^{3+}$ : ZBLAN waveguide laser. Optics Letters 2011;36:1587-9.

[4] Tan Y, Chen F, Vázquez de Aldana JR, Torchia GA, Benayas A, Jaque D. Continuous wave laser generation at $1064 \mathrm{~nm}$ in femtosecond laser inscribed $\mathrm{Nd}: \mathrm{YVO}_{4}$ channel waveguides. Applied Physics Letters 2010;97:031119.

[5] Siebenmorgen J, Petermann K, Huber G, Rademaker K, Nolte S, Tünnermann A. Femtosecond laser written stress-induced $\mathrm{Nd}: \mathrm{Y}_{3} \mathrm{Al}_{5} \mathrm{O}_{12}$ (Nd:YAG) channel waveguide laser. Applied Physics B 2009;97:251-5.

[6] Calmano T, Siebenmorgen J, Hellmig O, Petermann K, Huber. G. Nd:YAG waveguide laser with $1.3 \mathrm{~W}$ output power, fabricated by direct femtosecond laser writing. Applied Physics B 2010;100:131-5.

[7] Rodenas A, Kar. AK. High-contrast step-index waveguides in borate nonlinear laser crystals by 3D laser writing. Optics Express 2011:19:17820-33.

[8] Benayas A, Silva WF, Ródenas A, Jacinto C, Vázquez de Aldana J, Chen F, et al. Ultrafast laser writing of optical waveguides in ceramic Yb:YAG: a study of thermal and non-thermal regimes. Applied Physics A: Materials Science and Processing 2011;104:301-9.

[9] Ródenas A, Torchia GA, Lifante G, Cantelar E, Lamela J, Jaque F, et al. Refractive index change mechanisms in femtosecond laser written ceramic Nd:YAG waveguides: micro-spectroscopy experiments and beam propagation calculations. Applied Physics B 2009;95:85-96.

[10] Torchia GA, Rodenas A, Benayas A, Cantelar E, Roso L, Jaque D. Highly efficient laser action in femtosecond-written Nd:yttrium aluminum garnet ceramic waveguides. Applied Physics Letters 2008;92:111103.

[11] Dong N, Yao Y, Chen F, Vazquez de Aldana JR. Channel waveguides preserving luminescence features in $\mathrm{Nd}^{3+}: \mathrm{Y}_{2} \mathrm{O}_{3}$ ceramics produced by ultrafast laser inscription. Physica Status Solidi 2011:5:184-6.

[12] Fletcher LB, Witcher JJ, Troy N, Reis ST, Brow RK, Krol DM. Direct femtosecond laser waveguide writing inside zinc phosphate glass. Optics Express 2011;19:7929-36.
[13] Chen F, Vazquez de Aldana JR. Optical waveguides in crystalline dielectric materials produced by femtosecond-laser micromachining. Laser and Photonics Reviews 2013;1-26:1-25. http://dx.doi.org/10.1002/lpor.201300025.

[14] Okhrimchuk A, Mezentsev V, Dvoyrin V, Kurkov A, Sholokhov E, Turitsyn S et al. Waveguide-saturable absorber fabricated by femtosecond pulses in YAG: $\mathrm{Cr}^{4+}$ crystal for Q-switched operation of Yb-fiber laser. Optics Letters 2009;34:3881-3.

[15] Okhrimchuk A, Shestakov AV, Khrushchev I, Mitchell J. Depressed cladding, buried waveguide laser formed in a YAG: $\mathrm{Nd}^{3+}$ crystal by femtosecond laser writing. Optics Letters 2005;30:2248-50.

[16] Benayas A, Silva WF, Jacinto C, Cantelar E, Lamela J, Jaque F, et al. Thermally resistant waveguides fabricated in Nd:YAG ceramics by crossing femtosecond damage filaments. Optics Letters 2010;35:330-2.

[17] Siebenmorgen J, Petermann K, Huber G, Rademaker K, Nolte S, Tunnermann A Femtosecond laser written stress-induced $\mathrm{Nd}: \mathrm{Y}_{3} \mathrm{Al}_{5} \mathrm{O}_{12}(\mathrm{Nd}: \mathrm{YAG})$ channel waveguide laser. Applied Physics B 2009;97:251-5.

[18] Ren Y, Brown G, Ródenas A, Beecher S, Chen F, Kar AK. Mid-infrared waveguide lasers in rare-earth-doped YAG. Optics Letters 2012;37:3339-41.

[19] Jia Y, Chen F, Vázquez de Aldana JR. Efficient continuous-wave laser operation at $1064 \mathrm{~nm}$ in $\mathrm{Nd}: \mathrm{YVO}_{4}$ cladding waveguides produced by femtosecond laser inscription. Optics Express 2012;20:16801-6.

[20] Dong N, Chen F, Vazquez de Aldana JR. Efficient second harmonic generation by birefringent phase matching in femtosecond laser inscribed KTP cladding waveguides. Physica Status Solidi 2012;6:306-8.

[21] An Q, Ren Y, Jia Y, Vazquez de Aldana JR, Chen F. Mid-infrared waveguides in zinc sulfide crystal. Optical Materials Express 2013;3:466-71.

[22] Meyer JR, Hoffman CA, Bartoli FJ, Ram-Mohan LR. Type-II quantum-well lasers for the mid-wavelength infrared. Applied Physics Letters 1995;67:757-9.

[23] Vurgaftman I, Meyer JR, Tansu N, Mawst LJ. InP-based dilute-nitride midinfrared Type-II W quantum-well lasers. Journal of Applied Physics 2004;96:4653-5.

[24] Mawst LJ, Huang JYT, Xu DP, Yeh JY, Tsvid G, Kuech TF, et al. MOCVD grown dilute-nitride type-II quantum wells. IEEE Journal of Selected Topics in Quantum Electronics 2008;14:979-91.

[25] Bandyopadhyay N, Bai Y, Tsao S, Nida S, Slivken S, Razeghi M. Room temperature continuous wave operation of $\lambda: 3-3.2 \mu \mathrm{m}$ quantum cascade lasers. Applied Physics Letters 2012;101:241110.

[26] Kim S-S, Young C, Mizaikoff B. Miniaturized mid-infrared sensor technologies. Analytical and Bioanalytical Chemistry 2008;390:231-7.

[27] Mashanovich GZ, Milošević MM, Nedeljkovic M, Owens N, Xiong B, Teo EJ, et al. Low loss silicon waveguides for the mid-infrared. Optics Express 2011;19:7112-9.

[28] Milošević MM, Matavulj PS, Yang P, Bagolini A, Mashanovich GZ. Rib waveguides for mid-infrared silicon photonics. Journal of the Optical Society of America B 2009;26:1760-6.

[29] Maker AJ, Armani AM. Low-loss silica-on-silicon waveguides. Optics Letters 2011:36:3729-31.

[30] Ródenas A, Martin G, Arezki B, Psaila N, Jose G, Jha A, et al. Three-dimensional mid-infrared photonic circuits in chalcogenide glass. Optics Letters 2012;37:392-4

[31] 〈http://www.ispoptics.com〉.

[32] Lancaster DG, Gross S, Ebendorff-Heidepriem H, Fuerbach A, Withford MJ, Monro TM. $2.1 \mu \mathrm{m}$ waveguide laser fabricated by femtosecond laser directwriting in $\mathrm{Ho}^{3+}, \mathrm{Tm}^{3+}$ :ZBLAN glass. Optics Letters 2012;37:996-8.

[33] Liu H, Jia Y, Vázquez de Aldana JR, Jaque D, Chen F. Femtosecond laser inscribed cladding waveguides in Nd:YAG ceramics: fabrication, fluorescence imaging and laser performance. Optics Express 2012;20:18620-9.

[34] Okhrimchuk A, Mezentsev V, Shestakov A, Bennion I. Low loss depressed cladding waveguide inscribed in YAG:Nd single crystal by femtosecond laser pulses. Optics Express 2012;20:3832-43.

[35] RSoft Design Group, Computer Software BeamPROP, 〈http://www.rsoftdesign. com〉. 